Check for updates

Cite this: Nanoscale Adv., 2019, 1, 1200

\title{
In situ formation and superior lithium storage properties of tentacle-like ZnO@NCaCNTs composites $\uparrow$
}

\author{
Ying Wang, (D) a Shijia Fan, ${ }^{a}$ Fang Liao, ${ }^{a}$ Xinshi Zheng, ${ }^{a}$ Zhenguo Huang, ${ }^{d}$ \\ Yijing Wang $\left(\mathbb{D}{ }^{* b}\right.$ and Xiaopeng Han (D) *bc
}

\begin{abstract}
As a typical conversion-type electrode, $\mathrm{ZnO}$ has high theoretical $\mathrm{Li}^{+}$storage capacity and is low cost. However, its practical application is far away due to its limited rate performance and cycle stability. Herein, a novel structure of double carbon coated tentacle-like $\mathrm{ZnO}$ composite has been synthesized, which features in situ grown carbon nanotubes (CNTs) embedded in yolk-shell polyhedra, consisting of nitrogen-doped carbon layer (NC) coated $\mathrm{ZnO}$ nanoparticles (ZnO@NC(aCNTs). Excellent rate performance and good cycling stability are observed in the obtained ZnO@NC@CNTs, including a high reversible capacity of 800 and $617 \mathrm{~mA} \mathrm{~h} \mathrm{~g}^{-1}$ at 0.1 and $1.0 \mathrm{~A} \mathrm{~g}^{-1}$ and a low capacity decay of only $0.019 \%$ per cycle during 1000 cycles at $1.0 \mathrm{~A} \mathrm{~g}^{-1}$. The unique structure of this double carbon NC(aCNTs host can not only enhance electron transport throughout the whole electrode but also well accommodate the volume changes of $\mathrm{ZnO}$ during cycling, resulting in improved rate capability and cycle stability. In addition, the porous yolk-shell structure of the ZnO@NC@CNTs composite provides better contact between the electrolyte and active material, which enhances both capacity and rate performance of the electrode.
\end{abstract}

Received 20th September 2018

Accepted 3rd January 2019

DOI: $10.1039 / c 8 n a 00228 b$

rsc.li/nanoscale-advances

\section{Introduction}

Lithium ion batteries (LIBs) are widely employed in our daily life. Current portable high-performance electronic devices and electric vehicles constantly demand LIBs with higher energy/ power density, longer lifetime, and greater rate capability. However, commercialized graphite struggles to meet these requirements due to its low theoretical capacity $\left(372 \mathrm{~mA} \mathrm{~h} \mathrm{~g}^{-1}\right)$. To solve this issue, much effort has been devoted to the exploration of novel anode materials with high capacity, good cycle life, and satisfactory rate capability. ${ }^{1-9}$ Transition metal oxides (TMOs, $\mathrm{M}=\mathrm{Mn}, \mathrm{Fe}, \mathrm{Ni}, \mathrm{Zn}, \mathrm{Co}$, etc.), which have been widely used in other energy storage and conversion field such as sodium ion batteries ${ }^{10}$ and supercapacitors, ${ }^{11}$ have attracted extensive interest as promising anodes for LIBs due to their

${ }^{a}$ School of Chemistry \& Materials Science, Jiangsu Key Laboratory of Green Synthetic Chemistry for Functional Materials, Jiangsu Normal University, Xuzhou, Jiangsu 221116, China

${ }^{b}$ Key Laboratory of Advanced Energy Materials Chemistry (MOE), College of Chemistry, Nankai University, Tianjin 300071, China. E-mail: wangyj@nankai.edu.cn

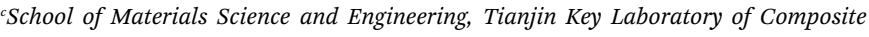
and Functional Materials, Tianjin University, Tianjin 300072, China. E-mail: xphan@tju.edu.cn

${ }^{d}$ School of Civil \& Environmental Engineering, University of Technology Sydney, NSW 2007, Australia

$\dagger$ Electronic supplementary information (ESI) available. See DOI: $10.1039 / \mathrm{c} 8 \mathrm{na} 00228 \mathrm{~b}$ high theoretical capacity and low cost. ${ }^{12-15}$ There are, however, a few drawbacks of TMOs as anode material in LIBs. First, large volume change occurs during the lithiation/delithiation cycles, leading to severe structural destruction and undesirable capacity loss. Second, poor electronic/ionic conductivity of the TMOs and their discharge mixture $\left(\mathrm{TM} / \mathrm{Li}_{2} \mathrm{O}\right)$ causes poor rate capability. To date, much effort has been devoted to improving the electrochemical performance of TMOs. ${ }^{16-18}$

Combining TMOs with conductive reinforcements (e.g., graphene, carbon nanotubes) is an effective way to make TMOs suitable for LIBs. Electrochemical performances of single carbon covered TMO hybrids with various morphologies have been reported, for example $\mathrm{C} @ \mathrm{SnO}_{2} @ \mathrm{C}$ nanofibers, ${ }^{19} \mathrm{MoO}_{2} @ \mathrm{C}$ nanorods, ${ }^{5} \mathrm{Co}_{3} \mathrm{O}_{4} @ \mathrm{NC}$ nanocages, ${ }^{20}$ and $\mathrm{ZnO} @ \mathrm{C}$ nanofibers. ${ }^{21}$ Recent theoretical and experimental results have demonstrated that metal-organic frameworks (MOFs) can also be used as templates and precursors to synthesize TMOs@carbon hybrids. ${ }^{22,23}$ For example, Yang et al. reported an IRMOF-1 derived ZnO@C hybrid, ${ }^{24}$ and our group reported a ZIF-67 derived $\mathrm{Co}_{3} \mathrm{O}_{4} @ \mathrm{NC}$ hybrid. ${ }^{20}$ These single carbon coated TMOs, however, usually suffer from limited interfacial conductivity due to the lack of conductive pathways throughout the electrode, because of which high utilization ratio of active materials is hard to obtain..$^{25}$ Therefore, it is very important to design a structure that features high integral structural stability, enhanced electron/ion diffusion pathways, and good conductivity (both local and interfacial). 
In this work, we have developed a novel structure of double carbon coated tentacle-like ZnO@NC@CNTs network, which has huge potential as an anode for high performance LIBs. As far as we know, this is the first time that in situ grown carbon nanotubes (CNTs) are coupled with N-doped carbon (NC) to form a double carbon host on a ZnO-based electrode. Specifically, the ZnO@NC@CNTs network is simply synthesized by annealing a Co-doped ZIF-8 precursor, without any separate carbon source. During the annealing process, organic linkers in the Co-doped ZIF-8 convert to a porous NC@CNT host that encapsulates $\mathrm{ZnO}$ nanoparticles (NPs) inside, forming ZnO@NC@CNTs with a yolk-shell architecture. The intimate contact between ZnO NPs and NC@CNTs effectively tolerates the volume change of $\mathrm{ZnO}$ during the charge/discharge process. The outstretched NC@CNTs provide multi-dimensional pathways for electron transportation throughout the whole electrode. With the synergistic effect of nitrogen doping, interconnected conductive tentacles, and macro-mesopores, the ZnO@NC@CNTs composite exhibited outstanding electrochemical performance, including excellent rate performance, high reversible capacity, and long cycle stability. The electrochemical performance observed here outperforms that of most of the other reported $\mathrm{ZnO}$-based electrodes, highlighting the structural advantages of this double carbon coated tentacle-like ZnO@NC@CNTs network.

\section{Experimental}

\section{Fabrication of ZIF-8 and Co-doped ZIF-8}

ZIF-8 was synthesized by using $\mathrm{Zn}\left(\mathrm{NO}_{3}\right)_{2} \cdot 6 \mathrm{H}_{2} \mathrm{O}$ and 2-methylimidazole (mIM) according to previous literature. ${ }^{26}$ The Codoped ZIF-8 was prepared via a cation exchange procedure: First, pure ZIF-8 powder was dispersed in $125 \mathrm{~mL}$ of methanol to form a $2 \mathrm{mg} \mathrm{mL}{ }^{-1}$ suspension. Then, $2.5 \mathrm{mM} \mathrm{Co}\left(\mathrm{NO}_{3}\right)_{2} \cdot 6 \mathrm{H}_{2} \mathrm{O}$ was mixed with the suspension and further aged for $24 \mathrm{~h}$. After several rinse-centrifugation cycles using ethanol and drying at $80{ }^{\circ} \mathrm{C}$ for $24 \mathrm{~h}$, the Co-doped ZIF-8 sample was obtained.

\section{Fabrication of ZnO@NC and ZnO@NC@CNTs networks}

A controllable two-step annealing process was used to transform the Co-doped ZIF-8 to ZnO@NC@CNTs. Typically, the Codoped ZIF-8 was first annealed at $550{ }^{\circ} \mathrm{C}$ in nitrogen for $2 \mathrm{~h}$ with a heating rate of $1{ }^{\circ} \mathrm{C} \mathrm{min}^{-1}$, and then subjected to another annealing step at $200{ }^{\circ} \mathrm{C}$ in air for $10 \mathrm{~h}$. For comparison, pure ZIF-8 was treated under the same conditions to get the ZnO@NC benchmark sample.

\section{Materials characterization}

X-ray diffraction (XRD, Bruker, D8-Advance), field-emission scanning electron microscopy (FESEM, JEOL, SU8010), transmission electron microscopy (TEM), high-resolution TEM (HR-TEM), selected area electron diffraction (SAED, performed on Tecnai G2 F20 S-TWIN), thermogravimetric analysis (TGA, TA-Q50), $\mathrm{N}_{2}$ adsorption/desorption isotherms (Quantachrome, Autosorb-IQ2-VP), X-ray photoelectron spectroscopy (XPS, Thermo ESCALAB 250XI), and Raman spectroscopy (Raman,
LabRAMHR800, $532 \mathrm{~nm}$ ) were employed for morphological and structural investigations.

\section{Electrochemical testing}

Electrochemical performance was evaluated by fabricating standard 2032 type coin cells, which were assembled in an argon-filled glove box with the oxygen and water content below 0.1 ppm. Typically, active materials, super conductive carbon black, and sodium carboxymethyl cellulose (CMC, Sigma Aldrich) were mixed in a weight ratio of $8: 1: 1$, dispersed in water, and then milled for $30 \mathrm{~min}$ to form a slurry. The slurry was cast onto copper foil using a doctor blade, and vacuum dried at $80{ }^{\circ} \mathrm{C}$ overnight. A lithium disc (MTI Corporation) was used as the counter electrode. $1 \mathrm{M} \mathrm{LiPF}_{6}$ (Sigma Aldrich) in ethylene carbonate (EC, Sigma Aldrich), diethyl carbonate (DEC, Alfa Aesar), and fluorinated ethylene carbonate (FEC, Sigma Aldrich) (volume ratio $6: 3: 1$ ) was used as the electrolyte. Polypropylene (PP, MTI Corporation) was used as the separator. Galvanostatic charge-discharge tests were carried out at room temperature on a battery testing system (LAND Wuhan, China) in a potential range of $0.01-3.00 \mathrm{~V}\left(v s\right.$. $\left.\mathrm{Li}^{-\mathrm{Li}^{+}}\right)$. Cyclic voltammetry (CV) tests and electrochemical impedance spectroscopy (EIS) measurements were performed on a CHI660E electrochemical work station.

\section{Results and discussion}

The preparation procedure of the tentacle-like ZnO@NC@CNTs network is illustrated in Fig. 1. The ion-exchange process is carried out between $\mathrm{Zn}^{2+}$ (in ZIF-8) and the added $\mathrm{Co}^{2+}$ to obtain Co-doped ZIF-8, which self-assembles as polyhedra with a coarse surface. During the first-step annealing in nitrogen, organic linkers in the Co-doped ZIF-8 decompose into amorphous N-doped carbon layers, covering the metallic NPs inside and forming a hierarchical yolk-shell morphology. Meanwhile, CNTs are in situ grown with the catalytic effect of Co. ${ }^{27-29}$ The second-step annealing is carried out in air to obtain fully oxidized ZnO@NC@CNTs. The cutaway view illustrates the yolk-shell structure of the ZnO@NC@CNTs networks.

No impurities are found in the full-scale XRD pattern of Co-doped ZIF-8 (compared with pure ZIF-8, Fig. S1a †), indicating

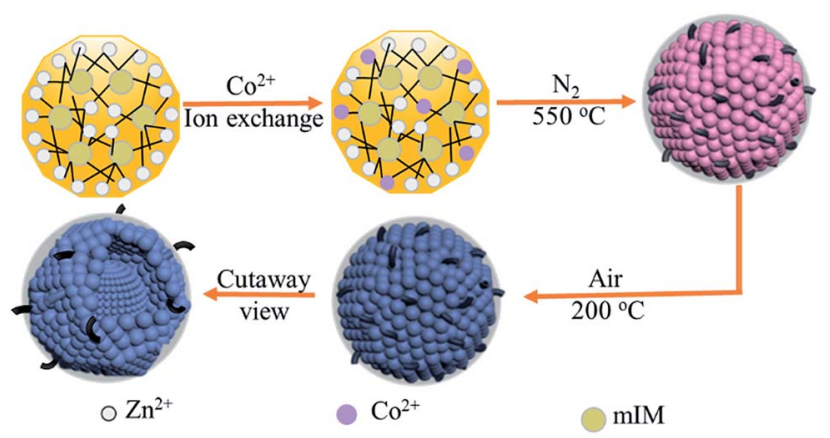

Fig. 1 Schematic illustration for the synthetic procedure of the ZnO@NCaCNTs composite. 
good structural preservation of the ZIF-8 phase after the ion exchange. Meanwhile, the partial magnification of the (110) peak is displayed in Fig. $\mathrm{S} 1 \mathrm{~b} \dagger$ to better present the peak shift. After the Co doping, a slight peak shift is observed, which is more obvious for the (110) peak, suggesting successful replacement of $\mathrm{Zn}$ by Co in the resulting sample. ${ }^{30,31}$ Both Co-doped ZIF-8 and ZIF-8 polyhedra show an average particle size of 400-450 nm (Fig. S2 $\dagger$ ). However, the surface of Co-doped ZIF-8 becomes quite coarse in comparison with the smooth surface of ZIF-8, implying the success of the ion exchange process on the surface. Thermal stability of the Co-doped ZIF-8 is characterized by TGA (Fig. S3 $\dagger$ ). Under the protection of $\mathrm{N}_{2}$, weight loss of the Co-doped ZIF-8 occurs between 470 and $650{ }^{\circ} \mathrm{C}$, corresponding to its structural decomposition. Therefore, $550{ }^{\circ} \mathrm{C}$ is selected as the first-step annealing temperature, during which organic linkers in the Codoped ZIF-8 convert into NC, gaseous products, or CNTs. The second-step annealing in air is used to ensure full oxidation of the resulting samples. SEM images of the pure ZIF- 8 derived ZnO@NC show the inheritance of the regular polyhedral outlines

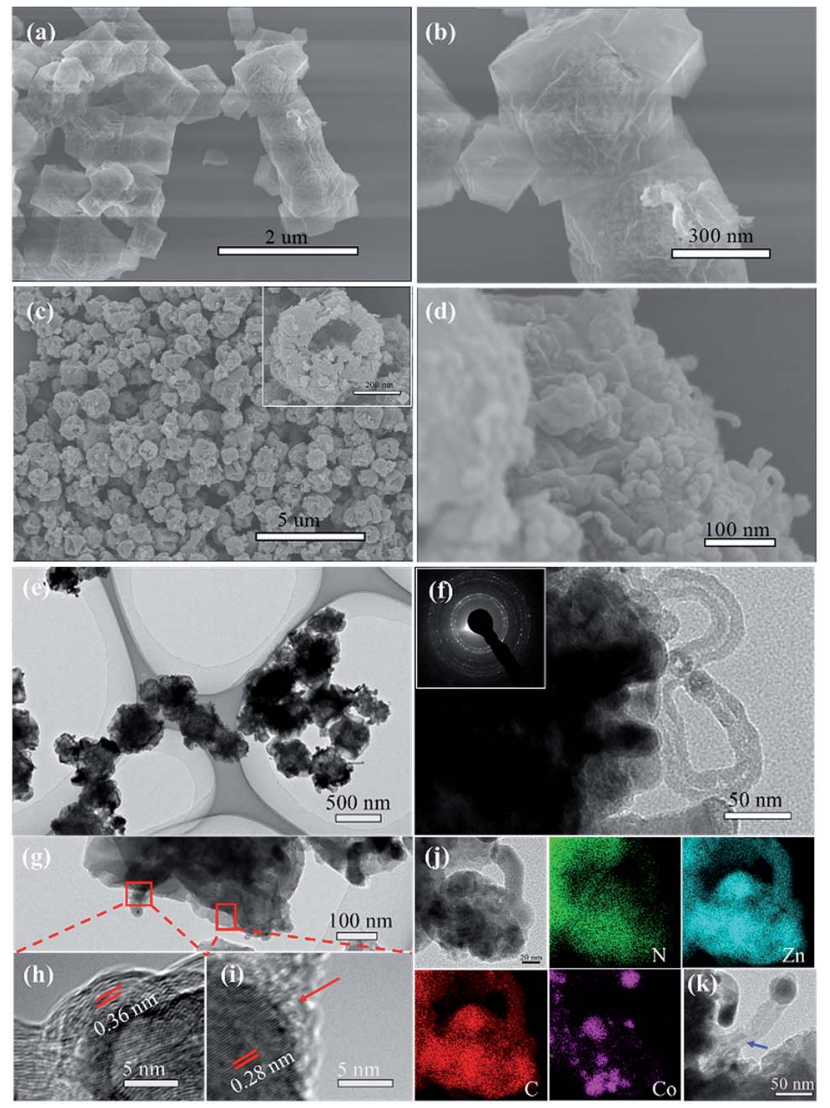

Fig. 2 SEM images of (a, b) ZnO@NC and (c, d) ZnO@NCaCNTs. (e-g) TEM images of theZnO@NC@CNTs network at different magnifications. (h, i) HR-TEM images of the ZnO@NC@CNTs at the selected area in (g). (j) Elemental mapping images of an individual ZnO@NC@CNTs polyhedron. Red, green, purple, and cyan are associated with carbon, nitrogen, cobalt, and zinc elements, respectively. (k) TEM picture of a selected CNT in the ZnO@NC(aCNTs. The inset of (c) is an enlarged picture of an individual ZnOaNC aCNTs polyhedron. The inset of $(f)$ is the SAED image. The red arrow in (i) and blue arrow in (k) point to amorphous carbon layers and $\mathrm{ZnO}$ inside the CNTs, respectively. from the precursor (Fig. 2a and b). The relatively irregular shape of ZnO@NC@CNTs is due to the formation of numerous CNTs on the rough surface (Fig. $2 c$ and d). A closer observation of the ZnO@NC@CNTs (inset in Fig. 2c) reveals a yolk-shell hierarchical structure that is composed of closely connected NPs as subunits. Interestingly, when increasing the Co content in Co-doped ZIF-8, CNTs are not observed in the derivatives (Fig. S4 $\dagger$ ). Therefore, the Co content is vital for the formation of CNTs. A smaller amount of Co acts as a catalyst to induce the growth of CNTs, while a higher Co content likely contributes to a quick formation of larger Co particles, which show a negligible catalytic effect. Effects of annealing temperature on the structure of ZnO@NC@CNTs were also investigated (Fig. S5†). Although the CNTs were also observed at a higher annealing temperature of $650{ }^{\circ} \mathrm{C}$, most polyhedra collapsed into irregular NPs, which may aggregate during long-term cycling.

TEM images of the ZnO@NC@CNTs further confirm the existence of CNTs with a diameter of about $15 \mathrm{~nm}$ (Fig. 2e-g). The contrast between the shell (dark) and the interior yolk (light) is consistent with the SEM observation. The SAED image of the ZnO@NC@CNTs (inset in Fig. 2f) exhibits a ring-like feature, which indicates a well crystallized structure, which is in agreement with the XRD result. The HR-TEM images (Fig. $2 \mathrm{~h}$ and i) show that the ZnO NPs are covered by amorphous carbon layers (red arrow) and further connected by CNTs. The graphitic interlayer spacing of $0.36 \mathrm{~nm}$ (Fig. 2h) corresponds to the (002) plane of the in situ formed CNTs. The Raman spectrum also confirms the existence of CNTs in ZnO@NC@CNTs (Fig. S6†). Generally, the D band (at $1316 \mathrm{~cm}^{-1}$ ) is associated with $\mathrm{sp}^{3}$-type disordered carbon, and the $\mathrm{G}$ band (at $1582 \mathrm{~cm}^{-1}$ ) derives from $\mathrm{sp}^{2}$-type graphitic carbon. ${ }^{32}$ The intensity ratio of the $\mathrm{D}$ to $\mathrm{G}$ band $\left(I_{\mathrm{D}} / I_{\mathrm{G}}\right)$ can be used to determine the graphitization degree of the material. Herein, the $I_{\mathrm{D}} / I_{\mathrm{G}}$ is calculated to be 0.4 , which indicated a higher graphitization degree. The lattice spacing of $0.28 \mathrm{~nm}$ (Fig. 2i) is related to the (100) plane of ZnO. The heterogeneous contraction caused the formation of yolk-shell structured ZnO@NC@CNTs. ${ }^{6,33,34}$ Elemental distribution is examined by TEM-EDS (Fig. 2j). Clearly, $\mathrm{C}$ and $\mathrm{N}$ co-exist and are uniformly distributed. Co exists predominantly at the edge of polyhedra and the tip of CNTs, confirming its catalytic effect on the growth of CNTs. ${ }^{32} \mathrm{Zn}$ is observed both in the polyhedra and along the CNTs. The blue arrow in Fig. $2 \mathrm{k}$ points to $\mathrm{ZnO}$ in the CNTs. Wang et al. observed a similar structure and the reason is ascribed to the restructuring of carbon. ${ }^{28}$

The XRD pattern of the ZnO@NC@CNTs shows main diffractions at $31.82,34.47,36.28,47.57,56.65,62.88,66.46$, 68.05, and 69.10 degrees (Fig. 3a), corresponding to the (100), (002), (101), (102), (110), (103), (200), (112), and (201) planes of ZnO (JCPDS: 36-1451), respectively. The absence of $\mathrm{Co} / \mathrm{CoO}_{x}$ diffractions in the XRD pattern is due to its low content (1.16 wt\%, Fig. S7 $\dagger$ ). The broad peak at $23.9^{\circ}$ is the carbon (002) plane. The carbon content in the ZnO@NC@CNTs is determined by TGA (Fig. 3b). In air, weight loss occurred between 400 and $525{ }^{\circ} \mathrm{C}$, corresponding to the decomposition of carbonaceous materials. Based on the TGA result, the amount of carbonaceous materials in ZnO@NC@CNTs is about $21 \mathrm{wt} \%$. Nitrogen isotherms of ZnO@NC@CNTs (Fig. 3c) exhibit typical 

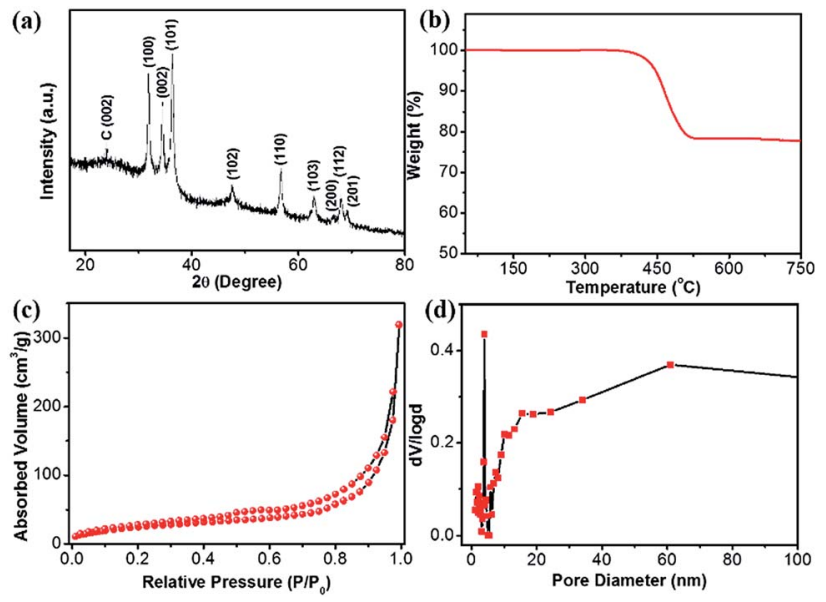

Fig. 3 (a) XRD, (b) TGA, (c) $\mathrm{N}_{2}$ isothermals, and (d) the corresponding pore-size distribution curve of the ZnO@NC@CNTs hybrid.

type IV curves with a H1 hysteresis loop, indicating the mesoporous nature. The narrow and small hysteresis loop in ZnO@NC@CNTs suggests the co-existence of macropores. The pore size distribution curve (Fig. 3d) reveals the existence of mesopores of $\sim 4.0 \mathrm{~nm}$ diameter and abundant macroporous structures, which are likely associated with the presence of CNTs and the voids between the yolk and shell. Notably, the combination of mesopores and macropores can facilitate better penetration of the electrolyte, which is important for enhancing the electrochemical performance. The specific surface area of ZnO@NC@CNTs is $151 \mathrm{~m}^{2} \mathrm{~g}^{-1}$, larger than that of $\mathrm{ZnO@NC}$ $\left(109 \mathrm{~m}^{2} \mathrm{~g}^{-1}\right.$, Fig. $\left.\mathrm{S} 8 \dagger\right)$.

The chemical composition and surface valence of the ZnO@NC@CNTs are investigated by an XPS technique (Fig. 4). The three peaks observed in the high-resolution $\mathrm{C}$ 1s spectrum at 284.6, 285.7, and $287.6 \mathrm{eV}$ can be assigned to the $\mathrm{sp}^{2} \mathrm{C}-\mathrm{C}$ bond, $\mathrm{sp}^{3} \mathrm{C}-\mathrm{C}$ bond, and $\mathrm{C}=\mathrm{O} / \mathrm{C}=\mathrm{N}$ groups (Fig. 4a), respectively. ${ }^{35}$ The $\mathrm{N}$ 1s spectrum confirms the formation of $\mathrm{C}-\mathrm{N}$ and
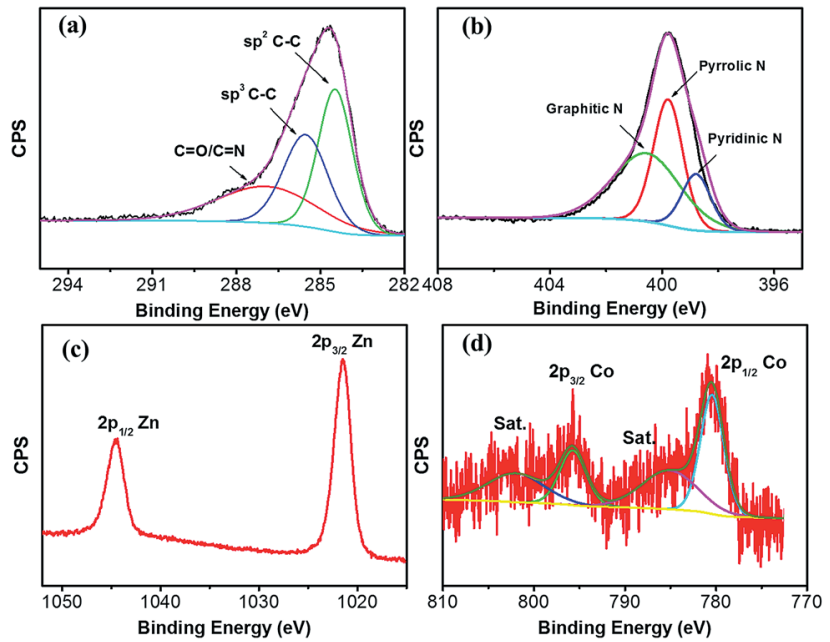

Fig. 4 High-resolution XPS spectrum of (a) C 1s, (b) N 1s, (c) Zn 2p, and (d) $\mathrm{Co} 2 \mathrm{p}$ of the ZnO@NCaCNTs hybrid.
$\mathrm{C}=\mathrm{N}$ bonds in the ZnO@NC@CNTs (Fig. 4b). Typical peaks are observed at 398.7, 399.8, and $400.7 \mathrm{eV}$, corresponding to pyridinic $\mathrm{N}$, pyrrolic $\mathrm{N}$, and graphitic $\mathrm{N}$, respectively. ${ }^{35,36}$ Generally, the N-doped carbon shows enhanced electronic conductivity and wettability compared to the non-doped counterparts. ${ }^{37}$ The $\mathrm{Zn} 2$ p spectrum shows two main peaks at 1045 and $1022 \mathrm{eV}$ (Fig. 4c), which are attributed to $\mathrm{Zn} 2 \mathrm{p}_{1 / 2}$ and $\mathrm{Zn}$ $2 \mathrm{p}_{3 / 2},{ }^{38,39}$ revealing the oxidation state of $\mathrm{ZnO}$. Fitting results of the Co 2p spectrum show two major peaks at 780.3 and $795.5 \mathrm{eV}$ (Fig. 4d). The $15.2 \mathrm{eV}$ energy difference between Co $2 \mathrm{p}_{1 / 2}$ and Co $2 \mathrm{p}_{3 / 2}$, together with the presence of the satellite peaks (marked as Sat.), is consistent with the oxidation state in $\mathrm{Co}_{3} \mathrm{O}_{4}{ }^{20,40}$

The unique structure of this ZnO@NC@CNTs is expected to benefit the lithiation/delithiation processes. Fig. 5a and b show cyclic voltammetry (CV) curves of the ZnO@NC and ZnO@NC@CNTs electrode for the initial five cycles. For the ZnO@NC@CNTs electrode, three broad peaks were observed at $1.04,0.57$ and $0.15 \mathrm{~V}$ in the first cathodic process, which are likely due to the formation of $\mathrm{Zn}-\mathrm{Li}$ alloys, reduction of $\mathrm{ZnO}$ to $\mathrm{Zn}$, and the growth of the solid electrolyte interface (SEI) film (caused by the partial decomposition of the carbonate electrolyte and Li salt), respectively. ${ }^{38,41}$ In the first anodic process, several successive oxidation peaks were observed $(0.3-0.8 \mathrm{~V})$, which could be assigned to the stepwise dealloying process of the $\mathrm{Li}-\mathrm{Zn}$ alloys (e.g., $\mathrm{LiZn}, \mathrm{Li}_{2} \mathrm{Zn}_{3}$, and $\left.\mathrm{LiZn}_{2}\right) .{ }^{21}$ The oxidation peak at $1.4 \mathrm{~V}$ is related to the decomposition of $\mathrm{Li}_{2} \mathrm{O}$ by reacting with $\mathrm{Zn} .{ }^{42}$ After the first scan, the major reduction peaks evolved into two peaks at about 0.33 and $0.55 \mathrm{~V}$, which can be assigned
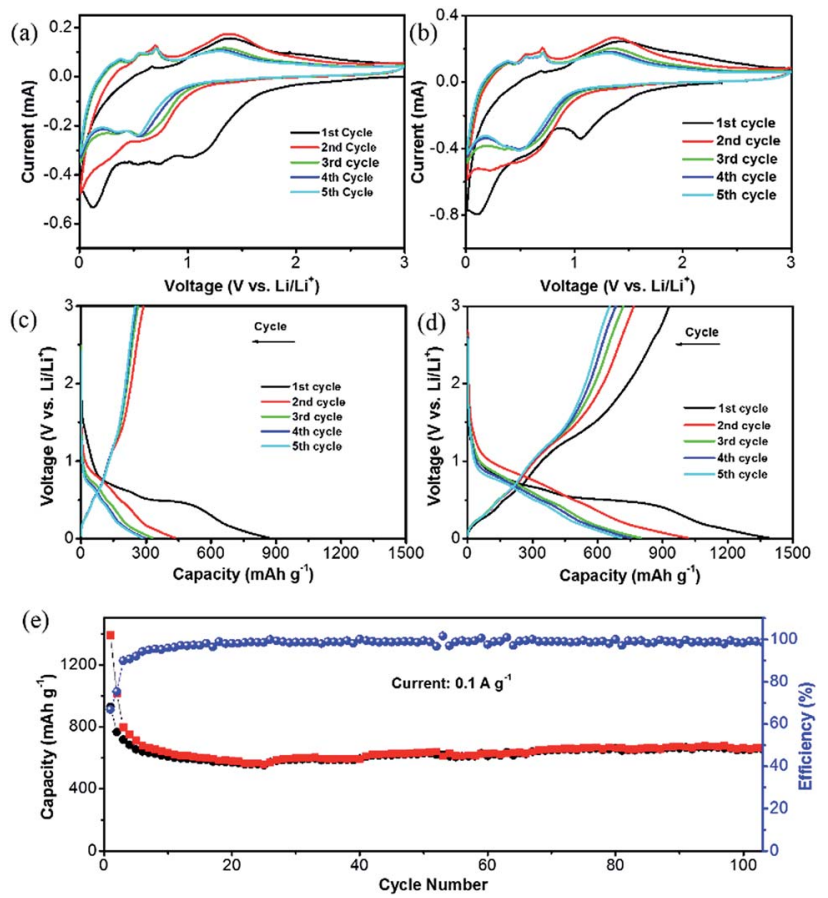

Fig. 5 Cyclic voltammograms of (a) ZnO@NC and (b) ZnO@NC@CNTs in the voltage window from 0.01 to $3.0 \mathrm{~V}\left(\mathrm{vs}\right.$. $\left.\mathrm{Li} / \mathrm{Li}^{+}\right)$at a scan rate of $0.5 \mathrm{mV} \mathrm{s}^{-1}$. Charge-discharge profiles of (c) ZnOaNC and (d) ZnO@NC@CNTs at a current density of $0.1 \mathrm{~A} \mathrm{~g}^{-1}$. (e) Cycle stability of the $\mathrm{ZnO}$ aNC@CNTs electrode at $0.1 \mathrm{~A} \mathrm{~g}^{-1}$. 
to the alloying process of Li-Zn. ${ }^{12,29,30}$ No significant difference was observed in the subsequent scans, indicating good reversibility of the redox process. Similar phenomena are observed in the ZnO@NC electrode, suggesting that the existence of CNTs did not alter its electrochemical mechanism.

These observations are further supported by the galvanostatic charge/discharge curves, which show a similar tendency (Fig. 5c and d). The high capacity of ZnO@NC@CNTs in the first cycle $\left(1390 \mathrm{~mA} \mathrm{~h} \mathrm{~g}^{-1}\right.$ ) is due to the reduction of $\mathrm{ZnO}$ and the formation of the SEI film. Capacity loss is observed in the initial few cycles, which then stabilized after the 3rd cycle (800 mA h g ${ }^{-1}$ for ZnO@NC@CNTs and $330 \mathrm{~mA} \mathrm{~h} \mathrm{~g}^{-1}$ for $\mathrm{ZnO}$ @NC). The capacity loss in the initial cycles is mainly caused by an incomplete conversion reaction and irreversible side reactions (e.g. formation of the SEI layer).$^{24}$ Although the initial coulombic efficiency (ICE) of the ZnO@NC@CNTs electrode $(66 \%)$ is higher than that of $\mathrm{ZnO} @ \mathrm{NC}$ and some of the electrodes in the literature, ${ }^{\mathbf{4 2 , 4 3}}$ such a value still cannot meet the requirements for practical applications, and further work should be carried out to improve the ICE, such as electrolyte optimization. As shown in Fig. 5e, the ZnO@NC@CNTs electrode exhibited a well-stabilized cycle performance at $0.1 \mathrm{~A} \mathrm{~g}^{-1}$. After 105 cycles, the ZnO@NC@CNTs can still deliver a high capacity of $672 \mathrm{~mA} \mathrm{~h} \mathrm{~g}^{-1}$ with a CE close to $100 \%$.

Fig. S9† compares the galvanostatic charge/discharge curves of the ZnO@NC and ZnO@NC@CNTs electrode at various current densities. The $\mathrm{ZnO@NC}$ electrode exhibited a low capacity of only $37 \mathrm{~mA} \mathrm{~g}^{-1}$ when the current increases to $1.0 \mathrm{~A} \mathrm{~g}^{-1}$. As expected, the rate performance is significantly improved for the ZnO@NC@CNTs sample. High reversible capacities of $800,617,409,356,294$, and $260 \mathrm{~mA} \mathrm{~h} \mathrm{~g}{ }^{-1}$ were delivered at $0.1,1.0,3.0,5.0,8.0$, and $10 \mathrm{Ag}^{-1}$, respectively. Even at ultra-high current densities of 15 and $20 \mathrm{~A} \mathrm{~g}^{-1}$, the ZnO@NC@CNTs electrode still maintains a remarkable capacity of 213 and $180 \mathrm{~mA} \mathrm{~h} \mathrm{~g}{ }^{-1}$, respectively. Such an extraordinary rate performance has been rarely reported among ZnO-based electrodes. ${ }^{11,24,28,30,31}$

To understand the excellent rate performance of ZnO@NC@CNTs, electrochemical impedance spectroscopy (EIS) studies are carried out (Fig. 6a). The semicircle in the Nyquist plots is attributed to the charge transfer resistance $\left(R_{\mathrm{ct}}\right)$, and the straight line is ascribed to the Warburg impedance $\left(W_{\mathrm{o}}\right)$, which is associated with the lithium ion diffusion process. Compared to ZnO@NC, the smaller semicircle of the ZnO@NC@CNTs electrode indicates faster reaction kinetics, which greatly contributes to its superior rate performance. The in situ generated conductive CNTs account for the improved electron transfer efficiency in the ZnO@NC@CNTs composite. The cycling performance of the ZnO@NC@CNTs electrode at different current densities is given in Fig. 6b. After cycling from 0.1 to $20 \mathrm{~A} \mathrm{~g} \mathrm{~g}^{-1}$ for 80 cycles, a high reversible capacity of $656 \mathrm{~mA} \mathrm{~h} \mathrm{~g}{ }^{-1}$ is still retained when the current reverses to $0.1 \mathrm{~A} \mathrm{~g}^{-1}$ at the 150th cycle, indicating good integrity of the active electrode. XRD and SEM images of the ZnO@NC@CNTs electrode after cycling are determined after fully charging to $3.0 \mathrm{~V}$ and then de-assembling in a glovebox. As shown in Fig. S10a, $\uparrow$ the typical diffraction peaks of $\mathrm{ZnO}$ are recovered
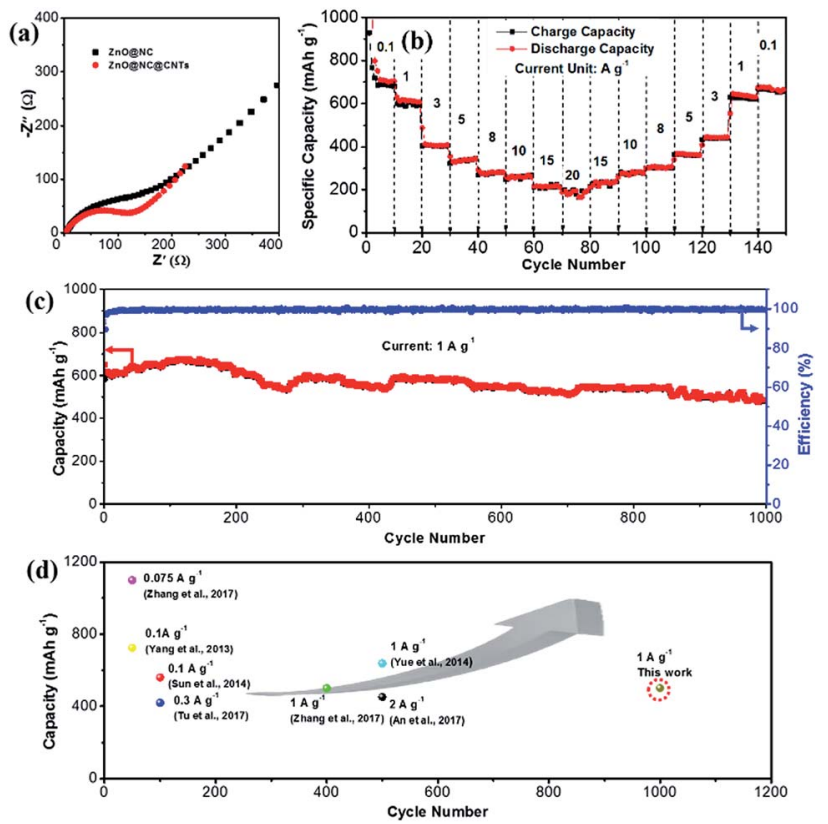

Fig. 6 (a) Nyquist plots of the ZnO@NC and ZnOaNCaCNTs electrodes. (b) Cycling performance at various current densities between 0.1 and $20 \mathrm{~A} \mathrm{~g} \mathrm{~g}^{-1}$ and (c) long-term cycle stability of the ZnO@NC@CNTs hybrid at $1.0 \mathrm{~A} \mathrm{~g}^{-1}$. (d) Electrochemical performance comparison of $\mathrm{ZnO}$ aNCaCNTs of this work with other $\mathrm{ZnO}$-based electrodes.

after the $\mathrm{Li}^{+}$insertion/extraction cycles, implying a full recovery of the $\mathrm{ZnO}$ phase. Compared with the initial electrode, a broad peak is observed at around $40^{\circ}$, which may be due to the decomposition of the electrolyte. The introduction of carbon black and CMC during the electrode assembling makes it difficult to observe the CNTs in the electrode after cycling (Fig. S10b †). Clearly, ZnO@NC@CNTs polyhedra still well embed in the electrode, and their unique morphology is maintained. All the above results confirm the remarkable durability of the ZnO@NC@CNTs electrode.

The long-term cycling performance of the ZnO@NC@CNTs is examined at $1.0 \mathrm{~A} \mathrm{~g}^{-1}$ (Fig. 6c). A high reversible capacity of $616 \mathrm{~mA} \mathrm{~h} \mathrm{~g}^{-1}$ is obtained at the beginning, which then gradually increases to a maximum value of $684 \mathrm{~mA} \mathrm{~h} \mathrm{~g}^{-1}$ at the 150th cycle. This phenomenon is generally observed in the TMO electrodes, which is due to gradual electrolyte wetting effects and/or the electrochemical activation of TMO electrodes. ${ }^{\mathbf{4 1}}$ After 1000 cycles, the electrode still delivered a high capacity of $501 \mathrm{~mA} \mathrm{~h} \mathrm{~g}^{-1}$, corresponding to only $0.019 \%$ capacity decay per cycle. Such extraordinary rate and cycling performances of ZnO@NC@CNTs are comparable to or even outperform those of other reported ZnO-based electrodes as displayed in Fig. 6d. ${ }^{4,45}$ To give a broader overview, electrochemical performance comparison of this novel ZnO@NC@CNTs electrode with that of other types of electrodes is also listed in Table S1, $\dagger$ which further confirms its structural superiority.

The excellent performance of the ZnO@NC@CNTs electrode likely originates from multiple factors. First, the double carbon structure of NC@CNTs covering the ZnO NPs forms a 3D 
tentacle-like conductive network, which facilitates the local and overall electron transportation and well accommodates the volume change of $\mathrm{ZnO}$ during cycling. Second, the combination of mesopores and macropores in the yolk-shell polyhedra can provide better contact between the electrolyte and electrode. Third, the hierarchical nanostructure shortens the diffusion distance for both $\mathrm{Li}^{+}$ions and electrons. These advantages synergistically result in the improved rate performance and cycle stability of the ZnO@NC@CNTs.

\section{Conclusions}

In summary, using Co-doped ZIF-8 as a precursor, a novel structure of NC@CNT double carbon coated tentacle-like ZnO composite has been synthesized. The ZnO@NC@CNTs electrode exhibits excellent Li-storage performance with a high reversible capacity ( $800 \mathrm{~mA} \mathrm{~h} \mathrm{~g}{ }^{-1}$ at $100 \mathrm{~mA} \mathrm{~g}^{-1}$ ), an excellent rate capability (capacity of $617,180 \mathrm{~mA} \mathrm{~h} \mathrm{~g}^{-1}$ at 1.0 and $20 \mathrm{~A} \mathrm{~g}^{-1}$ ), and good cycling stability (only $0.019 \%$ capacity loss per cycle during 1000 cycles at $1 \mathrm{~A} \mathrm{~g}^{-1}$ ). The superior electrochemical performance of ZnO@NC@CNTs confirms the structural advantages of this unique structure, which features an outstretched NC@CNT network and a yolk-shell hierarchal structure. This work may provide new insights for the fabrication of high-performance electrodes with integrated conductive carbon materials.

\section{Conflicts of interest}

There are no conflicts to declare.

\section{Acknowledgements}

This work was supported by the National Natural Science Foundation of China (21805117, 51602216), Jiangsu Province Science Foundation for Youths (BK20181014), National Natural Science of the Higher Education Institutions of Jiangsu Province (No. 18KJB150015), the Priority Academic Program Development Fund of Jiangsu Higher Education Institutions, and partially funded by the Australian Government through the Australian Research Council's Discovery Projects funding scheme (project DP170101773).

\section{Notes and references}

1 Y. Xu, Q. Li, H. Xue and H. Pang, Coord. Chem. Rev., 2018, 376, 292-318.

2 M. Zheng, S. Zhang, S. Chen, Z. Lin, H. Pang and Y. Yu, Nano Res., 2017, 10, 4305-4317.

3 T. B. Schon, B. T. McAllister, P. F. Li and D. S. Seferos, Chem. Soc. Rev., 2016, 45, 6345-6404.

4 V. Aravindan, Y. S. Lee and S. Madhavi, Adv. Energy Mater., 2015, 5, 1402225.

5 Y. Wang, Z. G. Huang and Y. J. Wang, J. Mater. Chem. A, 2015, 3, 21314-21320.
6 Y. Wang, B. F. Wang, F. Xiao, Z. G. Huang, Y. J. Wang, C. Richardson, Z. X. Chen, L. F. Jiao and H. T. Yuan, J. Power Sources, 2015, 298, 203-208.

7 Y. Wang, C. Y. Wang, H. N. Guo, Y. J. Wang and Z. G. Huang, RSC Adv., 2017, 7, 1588-1592.

8 Z. Zheng, Y. Zao, Q. Zhang, Y. Cheng, H. Chen, K. Zhang, M. S. Wang and D. L. Peng, Chem. Eng. J., 2018, 347, 563573.

9 Q. Zhang, H. Chen, L. Luo, B. Zhao, H. Luo, X. Han, J. Wang, C. Wang, Y. Yang, T. Zhu and M. Liu, Energy Environ. Sci., 2018, 11, 669-681.

10 H. Kim, H. Kim, Z. Ding, M. H. Lee, K. Lim, G. Yoon and K. Kang, Adv. Energy Mater., 2016, 6, 1600943.

11 Y. Yan, P. Gu, S. Zheng, M. Zheng, H. Pang and H. Xue, J. Mater. Chem. A, 2016, 4, 19078-19085.

12 J. Zhang, P. Gu, J. Xu, H. Xue and H. Pang, Nanoscale, 2016, 8, 18578-18595.

13 K. Q. Zhou, M. L. Kang, X. Q. He, Z. S. Hong, Z. G. Huang and M. D. Wei, J. Mater. Chem. A, 2017, 5, 18138-18147.

14 X. J. Gao, J. W. Wang, D. Zhang, K. Q. Nie, Y. Y. Ma, J. Zhong and X. H. Sun, J. Mater. Chem. A, 2017, 5, 5007-5012.

15 G. L. Xia, L. J. Zhang, F. Fang, D. L. Sun, Z. P. Guo, H. K. Liu and X. B. Yu, Adv. Funct. Mater., 2016, 26, 6188-6196.

16 G. Zhang, H. Zhang, X. Zhang, W. Zeng, Q. Su, G. Du and H. Duan, Electrochim. Acta, 2015, 186, 165-173.

17 Q. Xie, Y. Ma, X. Wang, D. Zeng, L. Wang, L. Mai and D. L. Peng, ACS Nano, 2016, 10, 1283-1291.

18 L. Wang, G. Zhang, Q. Liu and H. Duan, Mater. Chem. Front., 2018, 2, 1414-1435.

19 X. L. Wang, J. Z. Li, Z. Chen, L. J. Lei, X. P. Liao, X. Huang and B. Shi, J. Mater. Chem. A, 2016, 4, 18783-18791.

20 Y. Wang, C. Y. Wang, Y. J. Wang, H. K. Liu and Z. G. Huang, J. Mater. Chem. A, 2016, 4, 5428-5435.

21 G. H. An, D. Y. Lee and H. J. Ahn, ACS Appl. Mater. Interfaces, 2017, 9, 12478-12485.

22 H. Tang, M. Zheng, Q. Hu, Y. Chi, B. Xu, S. Zhang, H. Xue and H. Pang, J. Mater. Chem. A, 2018, 6, 13999-14024.

23 G. Zhang, S. Hou, H. Zhang, W. Zeng, F. Yan, C. C. Li and H. Duan, Adv. Mater., 2015, 27, 2400-2405.

24 S. J. Yang, S. Nam, T. Kim, J. H. Im, H. Jung, J. H. Kang, S. Wi, B. Park and C. R. Park, J. Am. Chem. Soc., 2013, 135, 73947397.

25 T. Jiang, F. Bu, X. Feng, I. Shakir, G. Hao and Y. Xu, ACS Nano, 2017, 11, 5140-5147.

26 N. L. Torad, M. Hu, Y. Kamachi, K. Takai, M. Imura, M. Naito and Y. Yamauchi, Chem. Commun., 2013, 49, 2521-2523.

27 N. Sikdar, B. Konkena, J. Masa, W. Schuhmann and T. K. Maji, Chem, 2017, 23, 18049-18056.

28 D. W. Su, M. Cortie and G. X. Wang, Adv. Energy Mater., 2017, 7, 1602014.

29 S. N. Zaretskiy, Y. K. Hong, D. H. Ha, J. H. Yoon, J. Cheon and J. Y. Koo, Chem. Phys. Lett., 2003, 372, 300-305.

30 W. Zhao, S. Wang, C. Feng, H. Wu, L. Zhang and J. Zhang, ACS Appl. Mater. Interfaces, 2018, 10, 40491-40499.

31 K. Zhang, M. Park, L. Zhou, G.-H. Lee, J. Shin, Z. Hu, S.-L. Chou, J. Chen and Y.-M. Kang, Angew. Chem., Int. Ed., 2016, 55, 12822-12826. 
32 J. Meng, C. Niu, L. Xu, J. Li, X. Liu, X. Wang, Y. Wu, X. Xu, W. Chen, Q. Li, Z. Zhu, D. Zhao and L. Mai, J. Am. Chem. Soc., 2017, 139, 8212-8221.

33 L. Huang, G. H. Waller, Y. Ding, D. C. Chen, D. Ding, P. X. Xi, Z. L. Wang and M. L. Liu, Nano Energy, 2015, 11, 64-70.

34 J. Guan, F. Mou, Z. Sun and W. Shi, Chem. Commun., 2010, 46, 6605-6607.

35 L. Zhang, Z. Su, F. Jiang, L. Yang, J. Qian, Y. Zhou, W. Li and M. Hong, Nanoscale, 2014, 6, 6590-6602.

36 F. Zheng, Y. Yang and Q. Chen, Nat. Commun., 2014, 5, 5261. 37 Y. Liu, N. Zhang, L. Jiao and J. Chen, Adv. Mater., 2015, 27, 6702-6707.

38 H. Zhang, Y. Wang, W. Zhao, M. Zou, Y. Chen, L. Yang, L. Xu, H. Wu and A. Cao, ACS Appl. Mater. Interfaces, 2017, 9, 37813-37822.

39 Y. Q. Zhang, Y. B. Lu, S. Feng, D. D. Liu, Z. L. Ma and S. Y. Wang, J. Mater. Chem. A, 2017, 5, 22512-22518.
40 Y. Q. Fan, N. Zhang, L. Y. Zhang, H. B. Shao, J. M. Wang, J. Q. Zhang and C. N. Cao, J. Electrochem. Soc., 2013, 160, F218-F223.

41 Z. H. Li, X. Yu, Y. Liu, W. X. Zhao, H. Zhang, R. M. Xu, D. H. Wang and H. Shen, J. Mater. Chem. A, 2016, 4, 19123-19131.

42 X. Sun, C. G. Zhou, M. Xie, H. T. Sun, T. Hu, F. Y. Lu, S. M. Scott, S. M. George and J. Lian, J. Mater. Chem. A, 2014, 2, 7319-7326.

43 Y. L. Zou, Z. G. Qi, Z. S. Ma, W. J. Jiang, R. W. Hu and J. L. Duan, J. Electroanal. Chem., 2017, 788, 184-191.

44 Y. Zhang, Y. Lu, S. Feng, D. Liu, Z. Ma and S. Wang, J. Mater. Chem. A, 2017, 5, 22512-22518.

45 H. Yue, Z. Shi, Q. Wang, Z. Cao, H. Dong, Y. Qiao, Y. Yin and S. Yang, ACS Appl. Mater. Interfaces, 2014, 6, 1706717074 . 